lution studies are probably necessary to solve the problem of the structure of the individual clouds and the temperature of the neutral hydrogen. Such high-resolution studies will also give the opportunity of studying more features in absorption and thus perhaps give some rough distance estimates. Clearly, we have made only the first step in the $21-\mathrm{cm}$ line research of the structure of our Galaxy.

\title{
20. HIGH-RESOLUTION STUDIES OF THE GALACTIC EQUATOR REGION
}

\section{F. J. KERR}

\section{CSIRO Radiophysics Laboratory, Sydney}

A large program of observations is being undertaken with the 210 -foot telescope at Parkes, to study the fine structure of the $21-\mathrm{cm}$ line emission in the region of the galactic equator. With the aid of a multi-channel receiver, $\mathrm{H}$-line profiles have been obtained every $6 \mathrm{~min}$ of arc (i.e. every half-beamwidth) along the equator from $l \mathrm{II}=300$ to $60^{\circ}$, and also from $b^{\mathrm{II}}=-3$ to $+3^{\circ}$ along a number of constantlongitude lines. The bandwidth of each channel of the receiver is equivalent to $8 \cdot 0$ $\mathrm{km} / \mathrm{sec}$, with a spacing of $7.0 \mathrm{~km} / \mathrm{sec}$ between the centres of adjacent channels. The data have been recorded on punched paper tape, and the main reduction is being carried out on the SILLIAC computer of the University of Sydney. A sample group of profiles, illustrating the great variability from point to point, has been published previously (Kerr 1962b).

In addition to the great increase in resolving power, the present survey has the advantage over the previous combined observations in that the regions north and south of the galactic centre are being studied with the same instrument and the same techniques. The very detailed results have been only partially reduced so far, but a number of conclusions can be presented.

\section{The Hydrogen Layer}

The thickness of the hydrogen layer between half-density levels can be derived from observations in the tangential-point regions. With the higher resolution, the thickness is found to be about $170 \mathrm{pc}$ in the region between 3 and $7 \mathrm{kpc}$ from the galactic centre, rather than the earlier $21-\mathrm{cm}$ value of $220 \mathrm{pc}$. The lower figure agrees better with values of layer thickness derived for cepheids and HII regions.

The new observations contirm the extreme flatness of the hydrogen layer, as indicated by the earlier lower-resolution surveys. Over the whole region inside a circle through the Sun, the deviations of the main layer from a plane are always less than a few tens of parsecs. A more precise solution for the galactic plane will result from the present survey.

\section{Detail in a Spiral Arm}

An interesting question connected with galactic spiral structure is the degree of continuity along an individual spiral arm. A detailed examination has been made of the "far outer arm", which is a well-marked feature on the far side of the Galaxy north of the centre. This arm was chosen for study because it can be seen from 
outside as an isolated entity, with a relatively small distance variation along its length. As shown in Figure 1 $(a)$, the variation in $21-\mathrm{cm}$ intensity seems to indicate that the arm consists of a series of patches, typically a few hundred parsecs across, rather than a smooth distribution of matter.

Other evidence indicates that this patchiness is probably a general property of spiral arms in the Galaxy, as indeed would be expected from the visual appearance of spiral features in other galaxies.
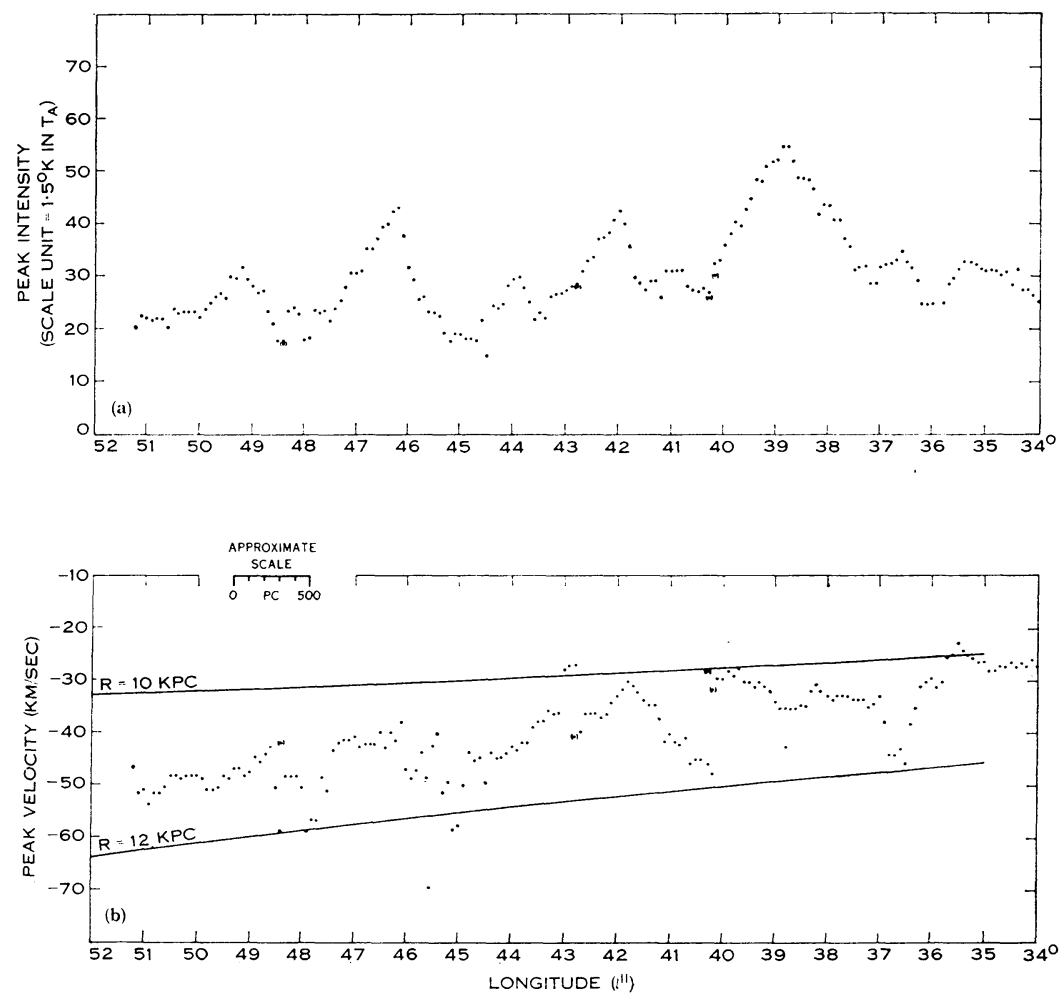

Fig. 1.-Variation of peak intensity and peak velocity along the far outer arm.

The velocity variation along the far outer arm also shows considerable fine structure. Two lines have been added to Figure $1(b)$ to indicate the velocities corresponding to circular motion at 10 and $12 \mathrm{kpc}$ from the centre. If all the gas is moving in circular orbits, the observed velocity fluctuation would represent a variation in $R$ over most of this range from 10 to $12 \mathrm{kpc}$, but such large spatial displacements along the arm seem unlikely. Some at least of the velocity fluctuation is probably due to local departures from circular motion.

\section{Rotation Curve}

A new rotation curve has been derived for each side of the galactic centre, by using as many of the observations (spaced 0.1 in $l^{\mathrm{II}}$ ) as were necessary to delineate the detail of each curve. In the galactic equator region, the line profile normally has a steep edge on one side - at positive velocities for northern longitudes, and 
negative velocities for southern longitudes. This part of the profile is due to gas at or near the tangential point (the point where the line of sight is tangential to a circle around the galactic centre); in this region the observed radial velocity gives the rotational velocity directly, if motion is assumed to be circular. In the present analysis, the velocity was read for the point halfway up the steep edge of the profile. This velocity is related to the velocity at the tangential point, but the true rotational velocity will be less by $15-20 \mathrm{~km} / \mathrm{sec}$ when the velocity dispersion of the gas clouds is allowed for by a method such as that of Kwee, Muller, and Westerhout (1954). Allowance has been made for the standard solar motion, following the usual practice.

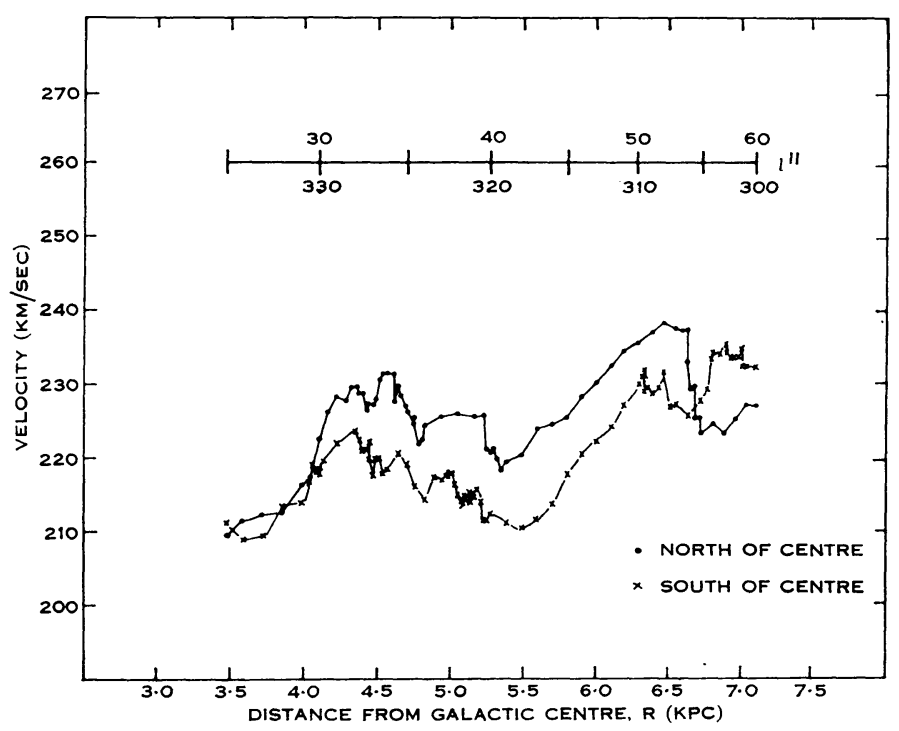

Fig. 2.-Galactic rotation curve for northern and southern sides of the centre. (These velocities correspond to points halfway up the steep edge of galactic equator profiles. The true rotational velocity is less by $15-20 \mathrm{~km} / \mathrm{sec}$.)

The results shown in Figure 2 confirm the apparent difference between the rotational velocities on the northern and southern sides of the centre. This difference has been interpreted (Kerr 1962a) as implying that material in the solar neighbourhood has a component of motion of several kilometres/second away from the galactic centre. An alternative possibility is that a real difference exists between the rotational velocities on the two sides; in this case, there must be a substantial mass asymmetry in the Galaxy, and orbits would not be circular.

The ups and downs of the rotation curves are believed to be related to the spiral pattern, with the higher parts of the curves indicating regions where spiral arms are being seen tangentially. A striking feature of Figure 2 is the high degree of similarity between the northern and southern curves, implying that the "spirals" are nearly circular in shape, and also that the two sides are rather symmetrical. The close similarity of the curves seems to argue against there being a real difference between the rotational velocities on the two sides, and in favour of an outward motion at the Sun producing an apparent difference. 


\section{Non-Circular Motion}

Much evidence is accumulating for non-circular motion in large regions of the Galaxy, but the earlier hypothesis of a general expansion of the gas throughout the Galaxy (Kerr 1962a) now appears to be an oversimplification. There seem to be some regions where motion is outward, and probably some where it is inward. A case where inward motion appears to be present is shown in Figure 3, which gives contours in velocity and latitude for a scan at $l \mathrm{II}=0.7$. There is a pronounced absorption dip near $b^{\mathrm{II}}=0^{\circ}$ at low positive velocities, associated with continuum radiation from a source which is believed to be near the centre. This radiation is apparently being absorbed by neutral hydrogen which is between the nucleus and the Sun and is moving away from the observer.

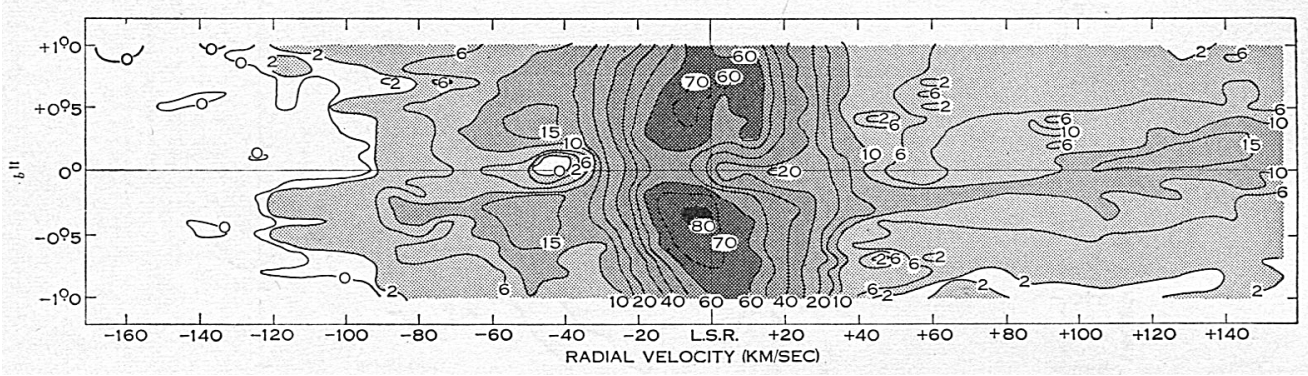

Fig. 3.-Contour diagram in the velocity-latitude plane for a scan at $l^{1 \mathrm{I}}=0^{\circ} \cdot 7$. (Scale unit $=1.5^{\circ} \mathrm{K}$ in $T_{A}$ ).

One of the primary objectives of the program is a very detailed spiral structure diagram, but it will be necessary to get a better understanding of the large-scale motions in the Galaxy before the distance problem can be satisfactorily solved.

With the high gain of the 210-foot telescope, it is possible to see absorption effects in several dozen places along the galactic equator, where continuum sources have been observed. These have not yet been examined in detail, but the results will be valuable as an additional aid in laying down a distance scale.

\section{References}

KERR, F. J. (1962a).-M.N. 123 : 327-45.

KerR, F. J. (1962b).-Sky and Tel. 24: 254-60.

Kwee, K. K., Muller, C. A., and Westerhout, G. (1954).-B.A.N. 12: 211-22.

\section{Discussion}

Oort: In the first place I wish to congratulate Dr. Kerr for the extremely interesting results he has been able to extract from the first period of observations with the Parkes telescope.

Referring to the absorption at positive velocities which Dr. Kerr finds at $l \mathrm{II}=0 \cdot 7$, I should like to ask whether one should conclude from this that we observe matter streaming toward the centre. Wouldn't this absorption be just what would be expected to result from the small rotating disk near the centre? The rotational velocities at $l \mathrm{II}=0 \cdot 7$ would certainly be large enough to account for the observed absorption.

Kerr: It appears too spread out in latitude for this ; however, a definite answer is not possible. I would like to anticipate a following session on the galactic centre by saying that the rotating disk appears to consist of many components. It may be a long time before a definite answer is possible. 
Oort: In spite of the many components one might expect the rotating matter to produce this absorption.

Kerr: This depends upon where the continuum source is in relation to the disk.

Oort: In connection with the tests for contraction or expansion of the Galaxy which Dr. Kerr mentioned, I should like to point to an investigation recently completed by Mr. Braes at Leiden. He used tracings made with the Dwingeloo telescope perpendicular to the galactic equator, in the region between $l^{I I}-5$ and $+5^{\circ}$. The outcome is that if there are general expansion or contraction effects they must be quite small, certainly less than $10 \mathrm{~km} / \mathrm{sec}$.

I would like to present some slides on the subject at a further session.

de Vaucouleurs: When we discuss expansion in the central regions we should keep in mind that the gas motion need not be isotropic in the plane.

Oort: What kind of gas? Spiral arms?

de Vaucouleurs : No, if there is any bar structure - even a weak one as in many galaxies one would rather expect any expansion motion to be mainly limited to gas streaming along the bar.

\section{LARGE-SCALE STRUCTURE AND KINEMATICS OF THE GALACTIC SYSTEM}

\section{B. LINDBLAD \\ Stockholms Observatorium}

It is, I believe, of fundamental importance to find out how far the structure and motions in the galaxies can be explained by gravitational theory. This does not deny that hydrodynamic and electromagnetic forces may play an important part. The special point which $I$ have in mind is the possibility, from the standpoint of gravitational theory, for the spiral structure to be a kind of quasi-steady state of comparatively long lifetime.

From the purely gravitational point of view we may expect that the spiral arms should be osculating to "dispersion orbits", that is orbits along which an association or a gaseous cloud has a natural tendency to disperse. An attempt to apply this criterion to the general spiral structure of the Galaxy has been made by B. Lindblad (1958). An application to the structure in the anticentre region has recently been made by Höglund (1963). Among more far-reaching attempts toward an interpretation of spiral structure may be mentioned the idea proposed by Aina Elvius (1958), later developed further by Aina Elvius and Herlofson (1960) and by Aina Elvius and P. O. Lindblad (1959), which considers the combined gravitational and electromagnetic force on a partially ionized gaseous ring and the effect of star formation releasing the electromagnetic force on the stellar matter. The gravitational theory of spiral structure has been developed extensively by P. O. Lindblad $(1960 a, b)$ who has shown by numerical computations that an ellipsoidal "dispersion ring" and bisymmetrical density waves in such a ring, which are proved to be in themselves steady phenomena, will produce perturbing effects by gravitational resonance leading to a development of spiral structure. This structure will in the first stages show "leading" arms with respect to the direction of rotation. At a later stage, however, these are transformed into arms of the "trailing" type. By the effects of differential rotation the arms will become successively more tightly wound, whereby the change 